CERN-TH 6702/92

\title{
Conformal Symmetry and Universal Properties of Quantum Hall States
}

\author{
Andrea CAPPELLI ${ }^{a *}$, Gerald V. DUNNE ${ }^{b}$, \\ Carlo A. TRUGENBERGER ${ }^{a}$ and Guillermo R. ZEMBA ${ }^{a}$ \\ a Theory Division**, C.E.R.N., 1211 Geneva 23, Switzerland \\ ${ }^{b}$ Dept. of Physics, University of Connecticut, 2152 Hillside Road, Storrs, CT 06268 USA
}

\begin{abstract}
The low-lying excitations of a quantum Hall state on a disk geometry are edge excitations. Their dynamics is governed by a conformal field theory on the cylinder defined by the disk boundary and the time variable. We give a simple and detailed derivation of this conformal field theory for integer filling, starting from the microscopic dynamics of $(2+1)$-dimensional non-relativistic electrons in Landau levels. This construction can be generalized to describe Laughlin's fractional Hall states via chiral bosonization, thereby making contact with the effective Chern-Simons theory approach. The conformal field theory dictates the finite-size effects in the energy spectrum. An experimental or numerical verification of these universal effects would provide a further confirmation of Laughlin's theory of incompressible quantum fluids.
\end{abstract}

CERN-TH 6702/92

October 1992

\footnotetext{
* On leave of absence from I.N.F.N., Firenze, Italy.

** Bitnets CAPPELLI, CAT, ZEMBA at CERNVM.
} 


\section{Introduction}

Laughlin's picture [1] of incompressible quantum fluids is the basis of our understanding of the plateaus in the quantum Hall effect (QHE) [2] [3] [4]. Planar electrons in a uniform magnetic field $B$ behave as a rigid droplet of liquid for specific rational values of the filling fraction $\nu=2 \pi \rho / B$ ( $\rho$ =density). For these values, the electrons assume a symmetric configuration with uniform density: excitations about such a configuration face an energy gap and hence the liquid is incompressible.

In systems with boundaries, as in the disk geometry we shall mostly consider here, the only possible low-energy excitations reside on the edge of the droplet. The edge can change shape at constant density, and thus the area of the droplet stays constant. This is the semiclassical picture for the infinite symmetry of the incompressible ground state under area-preserving diffeomorphisms [5] *

Starting with Halperin [7], a number of authors have been studying the dynamics of edge excitations [8] [9] [10] [11]. These live in one space dimension, the boundary of the circular droplet, and they are chiral due to the external magnetic field. For realistic samples, the radius $R$ of the circle is much larger than the typical microscopic scale given by the cyclotron radius $\ell=\sqrt{2 \hbar c / e B}$. Therefore, one can study the long-range effective theory of the edge excitations. This theory possesses universal properties which can be observed experimentally, as in the case of effective theories of critical phenomena.

In this theory, we are expecting a large symmetry connected to the already mentioned infinite symmetry of the ground state. Actually, Laughlin's theory [12] exhibits many indications of long-range coherence, which can be accounted for by the presence of a high degree of symmetry. The excitations are anyons, particles with fractional statistics [13]. Their wave-functions acquire non-trivial phases under mutual exchange, no matter what the separation distance [14]. This is indeed a consequence of infinite conformal invariance [15] of the chiral $(1+1)$-dimensional theory of the edge excitations.

Conformal invariance implies algebraic long-range order (power-law behaviour) in correlators of excitations localized around the edge. Precisely as in the low-temperature phase of the $X Y$ model, the long-range order should be understood in terms of conformal invariance and not of spontaneous symmetry breaking [16]. We believe that this point of view on long-range order is more natural than the one proposed in refs. [17.

* See also refs. [6] in connection with string theory. 
Previous studies of conformal invariance and edge excitations in the QHE were based mainly on the relation between Chern-Simons gauge theories and conformal field theories (CFT) [8] 10]. The bulk dynamics of an incompressible fluid can be described by an effective long-range field theory of an Abelian Chern-Simons gauge field. This field is the dual vector of the matter Hall current, while the Hall conductivity gives the value of the Chern-Simons coupling constant. On a disk geometry, the boundary gauge degree of freedom (a chiral bosonic field) becomes physical and describes the edge excitations in a bosonic language.

While this approach leads to an elegant formulation of the edge dynamics, the relation between the bosonic edge theory and the original problem of $(2+1)$-dimensional nonrelativistic electrons in Landau levels remains to be clarified. The key idea to this end was provided by Stone, who understood that the $(2+1)$-dimensional fermion goes into a $(1+1)$-dimensional relativistic chiral charged fermion (Weyl fermion), whose Dirac sea corresponds to the filled Landau level [9]. Here we refine Stone's idea by introducing a confining pressure via a novel procedure. For filling $\nu=1$, this allows a straightforward derivation of the fermionic boundary theory and its conformal symmetry directly from the microscopic physics of the Landau levels. We pay special attention to the details of the limiting procedure, including the role of boundary conditions. The resulting edge dynamics is a $c=1$ CFT describing a Weyl fermion with Neveu-Schwarz boundary conditions [18]. We also show how to deform the CFT in order to include the gaps for charged excitations on a disk geometry. Bosonization leads to the Floreanini-Jackiw model [19] of a chiral boson, which can be extended by introducing an additional coupling constant. By this procedure we make contact with the Chern-Simons approach. The simplicity of our limiting procedure leads to a clear picture of the interplay between $(2+1)$ - and $(1+1)$-dimensional physics.

While confirming the results of previous investigations [8] [9] [10] about the charge and statistics of excitations, we stress the role of finite-size effects in the physics of the QHE. Indeed, conformal invariance on the cylinder (the spatial boundary circle times the time coordinate) implies a non-trivial result [20]: the $O(1 / R)$-terms ( $R$ being the radius of the droplet) in the energy spectrum are universal. These include both the Casimir groundstate energy and the $1 / R$-corrections to the gaps of excitations. The former represents a quantum pressure of the electron liquid and is determined by the central charge $c$ of the CFT. The latter are determined by the conformal dimensions of the primary fields.

The results of conformal invariance for charge and statistics of excitations are in total agreement with Laughlin's theory and can be considered an a posteriori justification of this 
theory based on symmetry principles. The $1 / R$-terms in the energy spectrum are related to the charge and statistics of the excitations. Therefore, their comparison with experiments and numerical simulations would provide a direct confirmation of Laughlin's theory. Moreover, the $1 / R$-spectroscopy of hierarchical states is a promising tool for uncovering their dynamics.

Our straightforward relation between boundary and bulk theories also helps understanding some works which developed a formal analogy between conformal correlators and the Laughlin wave function [21] [22] [23] [24]. In short, the conformal correlators are defined on the boundary circle, but also determine the analytic part of correlators in the plane by analytic continuation. Therefore, most of the results obtained from the formal analogy are actually correct. The "topological order" 25] of the Laughlin wave function on a toroidal geometry is due to a well-known interplay between conformal and modular invariance on the torus [20], which has been discussed specifically in refs. [23] [26].

The outline of the paper is as follows. In section 2, we review the Landau level problem in the case of filling fraction $\nu=1$, and modify the Hamiltonian so as to include the boundary effects in a realistic finite sample. In section 3 , we discuss the thermodynamic limit $R \rightarrow \infty$, and derive the $(1+1)$-dimensional theory of the Weyl fermion and its conformal invariance. In section 4, we discuss the physical consequences of this theory and the universal finite-size effects of the quantum Hall system. In section 5, the bosonization of the Weyl fermion is carried out, by using the canonical theory of the chiral boson. In section 6 , this theory is used to describe the edge excitations of the simplest fractional Hall states. A more technical result is presented in Appendix A. This reports a direct derivation of long-range order in the density matrix on the boundary. 


\section{Integer Quantum Hall state for a finite sample}

\subsection{Landau levels}

We consider spin-polarized, planar electrons of mass $m$ and electric charge $e$ in an external, uniform, magnetic field $B>0$ (units $\hbar=1, c=1$ ). The one-particle Hamiltonian is given by

$$
H=-\frac{1}{2 m}(\nabla-i e \mathbf{A})^{2}-\frac{e B}{2 m},
$$

where the second term represents the Pauli interaction. Since we shall consider circular geometries, we choose to use the the symmetric gauge $A_{i}=\frac{B}{2} \varepsilon^{i j} x^{j}, \quad i, j=1,2$, for the external vector potential. The fundamental scale set by the external magnetic field is the magnetic length, $\ell=\sqrt{2 / e B}$. In the following, we will make extensive use of the complex notation $z=x^{1}+i x^{2}, \bar{z}=x^{1}-i x^{2}, \partial=\partial / \partial z, \bar{\partial}=\partial / \partial \bar{z}$. By introducing two commuting sets of harmonic oscillator operators

$$
\begin{aligned}
d=\frac{z}{2 \ell}+\ell \bar{\partial}, & d^{\dagger}=\frac{\bar{z}}{2 \ell}-\ell \partial, & & {\left[d, d^{\dagger}\right]=1, } \\
c=\frac{\bar{z}}{2 \ell}+\ell \partial, & c^{\dagger}=\frac{z}{2 \ell}-\ell \bar{\partial}, & & {\left[c, c^{\dagger}\right]=1, }
\end{aligned}
$$

the Hamiltonian (2.1) and the canonical angular momentum $J=-i x^{i} \varepsilon^{i j} \partial_{j}$ can be rewritten as

$$
\begin{aligned}
& H=\omega d^{\dagger} d, \\
& J=c^{\dagger} c-d^{\dagger} d,
\end{aligned}
$$

where $\omega=e B / m$ is the cyclotron frequency. Since the operators $c$ and $d$ commute, the spectrum consists of infinitely degenerate levels of energy $\varepsilon_{n}=\omega n$ : these are called the Landau levels. The degenerate states in one Landau level are characterized by the angular momentum eigenvalue $l$. Wave functions $\psi$ of the first Landau level satisfy $d \psi=0$ and have vanishing energy due to the additional Pauli interaction in (2.1). A complete basis is given by

$$
\psi_{l}(z, \bar{z})=\frac{1}{\ell \sqrt{\pi}} \frac{1}{\sqrt{l !}}\left(\frac{z}{\ell}\right)^{l} \mathrm{e}^{-|z|^{2} / 2 \ell^{2}} .
$$

Note that the radial part of these wavefunctions is sharply peaked around a radius $r_{l}=\ell \sqrt{l}$. Higher Landau levels basis states can be obtained by repeated action of the operators $d^{\dagger}$ 
and $c^{\dagger}$ on $\psi_{0}$ :

$$
\begin{aligned}
\psi_{n, l}(z, \bar{z}) & =\frac{\left(c^{\dagger}\right)^{l+n}}{\sqrt{(l+n) !}} \frac{\left(d^{\dagger}\right)^{n}}{\sqrt{n !}} \psi_{0}(z, \bar{z}) \\
& =\frac{1}{\ell} \sqrt{\frac{n !}{\pi(l+n) !}}\left(\frac{z}{\ell}\right)^{l} L_{n}^{l}\left(\frac{|z|^{2}}{\ell^{2}}\right) \mathrm{e}^{-|z|^{2} / 2 \ell^{2}}
\end{aligned}
$$

Here $n=0,1, \ldots$ labels the energy level, $l+n \geq 0$, and $L_{n}^{l}(x)$ are the generalized Laguerre polynomials [27].

The many-body problem of $N$ electrons is described, in second quantization, by the Hamiltonian

$$
H=\frac{1}{2 m} \int d^{2} \mathbf{x}\left(D_{i} \Psi\right)^{\dagger}\left(D_{i} \Psi\right)-\frac{1}{2 m} \int d^{2} \mathbf{x} e B \rho
$$

with $D_{i}=\partial_{i}+i e A_{i}$ the covariant derivative and $\rho(\mathbf{x}, t)=\Psi^{\dagger}(\mathbf{x}, t) \Psi(\mathbf{x}, t)$ the particlenumber density. The field operator $\Psi(\mathbf{x}, t)$ possesses an expansion in terms of the singleparticle energy and angular momentum eigenstates $\psi_{n, l}(\mathbf{x})$

$$
\Psi(\mathbf{x}, t)=\sum_{n=0}^{\infty} \sum_{l=-n}^{\infty} a_{l}^{(n)} \psi_{n, l}(\mathbf{x}) e^{-i n \omega t}
$$

The coefficients are fermionic Fock annihilators and creators satisfying

$$
\left\{a_{k}^{(n)}, a_{l}^{(m) \dagger}\right\}=\delta_{n, m} \delta_{k, l}
$$

with all other anticommutators vanishing. In the following we will consider mostly the physics of the first Landau level, for which the field operator takes the (time-independent) form

$$
\Psi(\mathbf{x})=\sum_{l=0}^{\infty} a_{l} \psi_{l}(\mathbf{x})
$$

with $\psi_{l}(\mathbf{x})$ given by (2.4) and $a_{l} \equiv a_{l}^{(0)}$. The property $d \psi_{l}=0$ can be also written as a self-dual condition* on the field operator

$$
D_{+} \Psi(\mathbf{x}) \equiv\left(D_{1}+i D_{2}\right) \Psi(\mathbf{x})=0 .
$$

* The corresponding condition $D_{-} \Psi=0$ would hold for an external magnetic field with the opposite sign. 


\subsection{Incompressible ground state}

In the following we are interested in universal, long-range properties of the many-body state consisting of one completely filled Landau level on a disk geometry. By this we mean a state $|\Omega\rangle$ in which all angular momentum states of the first Landau level are occupied up to (and including) a maximal angular momentum $L$ (the total number of particles is then $N=L+1)$ :

$$
|\Omega\rangle=a_{0}^{\dagger} a_{1}^{\dagger} \ldots a_{L}^{\dagger}|0\rangle
$$

where $|0\rangle$ is the Fock vacuum. Since the single-particle angular momentum states are peaked around radii $r_{l}=\ell \sqrt{l}$, the state under consideration consists of a circular droplet of radius approximately given by $R \simeq \ell \sqrt{L}$, as is rendered pictorially in Fig. 1. This configuration is clearly incompressible: a compression of the droplet would lower its total angular momentum and face an energy gap $\omega$, since at least one electron would be promoted to the next Landau level. The incompressibility is reflected in the spatial distribution of the matter. The expectation value of the density is readily computed in the second-quantized formalism,

$$
\langle\Omega|\rho(\mathbf{x})| \Omega\rangle=\frac{1}{\ell^{2} \pi} \mathrm{e}^{-r^{2} / \ell^{2}} \sum_{l=0}^{L} \frac{1}{l !}\left(\frac{r}{\ell}\right)^{2 l}
$$

and is shown in Fig. 2. It is constant for $r \ll \ell \sqrt{L}$, and drops rapidly to zero around $r \simeq \ell \sqrt{L}$. Thus incompressibility implies uniform density. Another quantity of interest is the expectation value of the current

$$
J^{i}(\mathbf{x})=\frac{1}{2 i m}\left\{\Psi^{\dagger}(\mathbf{x}) D_{i} \Psi(\mathbf{x})-\left(D_{i} \Psi(\mathbf{x})\right)^{\dagger} \Psi(\mathbf{x})\right\}
$$

This is easily obtained by combining (2.13) with $\partial_{i} \rho=\Psi^{\dagger} D_{i} \Psi+\left(D_{i} \Psi\right)^{\dagger} \Psi$ and using the self-dual condition (2.10). This gives

$$
\left\langle\Omega\left|J^{i}(\mathbf{x})\right| \Omega\right\rangle=-\frac{1}{2 m} \varepsilon^{i j} \partial_{j}\langle\Omega|\rho(\mathbf{x})| \Omega\rangle
$$

which is shown in Fig. 3. The current vanishes in the interior, where the density is constant; however, the state supports a transverse, circular current around the boundary of the droplet. This is the edge current originally discussed by Halperin [7] and gives a first indication that non-trivial dynamics is concentrated around the boundary. This boundary dynamics will be studied in detail in the following. 
We must distinguish between bulk and boundary interactions. Here we are not interested in the bulk dynamics which creates the incompressible ground state. To understand this dynamics, one should consider the microscopic interaction of electrons with impurities and their Coulomb interaction, and then solve the strongly interacting problem. Rather, we are going to assume that the incompressible ground state is indeed formed. This corresponds to an equilibrium configuration for the bulk interactions with a gap for density fluctuations. Therefore, it is reasonable to neglect these in the study of long-wavelength edge fluctuations.

The boundary interaction is described by a finite-size modification of the one-body Hamiltonian of Landau levels. This modification can be obtained by quantization in a finite disk (or annulus) geometry (Dirichlet boundary conditions) [7]. However, physically, it originates from the external potential due to the neutralizing background which keeps the electrons together [12]. Both effects lift the degeneracy of the Landau levels near the boundary. The angular momentum eigenstates in the first Landau level acquire a spectrum $\varepsilon_{l}$ with $\Delta \varepsilon_{l} / \Delta l>0$ for $l \sim L$. As a consequence, also decompressions, i.e., transitions to states with higher angular momentum, cost energy and $|\Omega\rangle$ is the unique ground state. Typically, the cost in energy for transitions of a few electrons from states of angular momentum $L-\delta l$ to states of angular momentum $L+\delta l$, with $\delta l \ll L$, is much smaller than the cyclotron energy. These edge excitations constitute the generic low-lying excitations about the incompressible state $|\Omega\rangle$.

For $L \gg 1$ the spectrum $\varepsilon_{l}$ can be taken as approximately linear in a region around $L:$

$$
\varepsilon_{l}=\alpha(l-L)+\beta
$$

Since this spectrum originates dynamically from the neutralizing background, the coefficients $\alpha$ and $\beta$ depend generically on the particle number $N=L+1$. In particular, one can consider their expansions in powers of $\sqrt{L} \simeq R / \ell$, where $R$ is the size of the system. As we now show, the dynamics of edge excitations is governed by a conformal field theory if the region of approximately linear spectrum increases as $L \rightarrow \infty$ and if $\alpha$ possesses an expansion

$$
\alpha=\frac{v}{\ell \sqrt{L}}+O\left(\frac{1}{L}\right)
$$

Here $v$ is the "velocity of light" of the emerging relativistic theory with spectrum $\varepsilon \propto v p$ with $p=l / \ell \sqrt{L}$ the one-dimensional momentum. Taking into account the general expansion of $\beta$,

$$
\beta=\beta_{0}-\frac{v \mu}{\ell \sqrt{L}}+O\left(\frac{1}{L}\right)
$$


the spectrum $\varepsilon_{l}$ up to order $O(1 / \sqrt{L})$ takes the form

$$
\varepsilon_{l}=\beta_{0}+\frac{v}{\ell \sqrt{L}}(l-L-\mu)
$$

with $\mu$ a dimensionless parameter playing the role of a chemical potential.

At this point we must distinguish between a geometry with two boundaries, i.e. an annulus, and the disk geometry with only one boundary. On an annulus geometry, the parameter $\beta_{0}$ becomes irrelevant for all edge excitations since the spectrum $\varepsilon_{l}$ takes the generic form shown in Fig. 4. Indeed, by considering $L_{1}, L_{2} \gg 1, L_{2} / L_{1}=\lambda=O(1)$, stability requires that the Fermi levels at the two edges can differ at most by terms of $O\left(1 / \ell \sqrt{L_{2}}\right)$. Therefore, one can reabsorbe $\beta_{0}$ by an overall shift in the zero of energy, thereby obtaining the spectra

$$
\varepsilon_{l}=\left\{\begin{aligned}
-\frac{v_{1}}{\ell \sqrt{L_{1}}}\left(l-L_{1}+\mu_{1}\right), & l \simeq L_{1}, \\
\frac{v_{2}}{\ell \sqrt{L_{2}}}\left(l-L_{2}-\mu_{2}\right), & l \simeq L_{2} .
\end{aligned}\right.
$$

At each edge there are two types of excitations: neutral particle-hole excitations and charged excitations which amount to a transfer of particles from one edge to the other. Both are gapless since charge transfer costs only $O\left(1 / \ell \sqrt{L_{2}}\right)$ energy. As we now show, in the thermodynamic limit $L_{1}, L_{2} \rightarrow \infty$ all these excitations span the Hilbert space of a $c=1 \mathrm{CFT}$. This is also the picture that emerges from the effective Chern-Simons gauge theory approach [8] [10].

The situation is different on a disk geometry. Here the generic spectrum $\varepsilon_{l}$ looks as in Fig. 5 and it costs a finite amount of energy to produce charged edge excitations, since one has to transfer charge between the edge and the center of the disk. A positive charge on the edge corresponds to a quasi-hole in the center of the disk and costs an energy $\beta_{0}+O(1 / \ell \sqrt{L})$. A negative charge on the edge corresponds to a quasi-particle at the center of the disk and costs an energy $\Delta-\beta_{0}+O(1 / \ell \sqrt{L})$, where $\Delta$ is the gap to the next energy band (for $\nu=1$ we have e.g. $\Delta=\omega$ ). The gapfulness of the charged excitations spoils conformal invariance. However, this is not a fatal problem; as we will show, the $c=1$ CFT is still relevant insofar as it predicts the universal finite-size corrections to the gaps and the static properties of correlators on the edge. 


\subsection{Confining pressure via a boundary Hamiltonian}

In order to discuss quantitatively the effects described above, we introduce a confining pressure by a novel procedure, which also leads automatically to the mapping on a relativistic CFT. We pick a circle of radius $R$ in the infinite plane and we define a new Hamiltonian $H_{R}$ as

$$
H_{R}=\frac{1}{2 m} \int_{R} d^{2} \mathbf{x}\left(D_{i} \Psi\right)^{\dagger}\left(D_{i} \Psi\right)-\frac{1}{2 m} \int_{R} d^{2} \mathbf{x} e B \rho
$$

where the subscript $R$ denotes integration of the radial coordinate only up to $R$. While being defined in the infinite plane, the new Hamiltonian mimics the system in a disk of radius $R$. In this finite geometry, the degeneracy of the lowest Landau level is given by the magnetic flux in quantum units through the disk, $\Phi \equiv R^{2} / \ell^{2}$. In the plane and for integer filling $\nu=1,(\Phi+1)$ electrons occupy approximatively a circular region of radius $R$ (see fig. 2). We thus relate the number of particles $L+1$ to the radius $R$ as follows

$$
\Phi=\frac{R^{2}}{\ell^{2}}=L+\mu, \quad L \gg 1, \mu=O(1)
$$

After this identification, let us show that $H_{R}$ correctly reproduces the realistic spectrum descibed in the previous section. Note that the eigenfunctions $\psi_{l}(\mathbf{x})$ of the first Landau level in the plane still diagonalize $H_{R}$ by angular integration. The lowest Landau level Fock space is thus unchanged; however the degeneracy is lifted and we obtain the one-particle spectrum

$$
\varepsilon_{l}=\frac{\omega}{2} \frac{\Phi^{l} e^{-\Phi}}{l !}(l-\Phi) .
$$

The field operator restricted to the first Landau level $\Psi$ in eq.(2.9) acquires a time dependence:

$$
\Psi(\mathbf{x}, t)=\sum_{l=0}^{\infty} a_{l} \psi_{l}(\mathbf{x}) e^{-i \varepsilon_{l} t}
$$

The spectrum in eq.(2.22) is shown in Fig. 6 for $\Phi=10$; it becomes approximately linear for $\Phi-\sqrt{\Phi} \leq l \leq \Phi+\sqrt{\Phi}$. The minimum and maximum are located at $\Phi \pm \sqrt{\Phi}$, respectively, and the maximal value of $\varepsilon_{l}$ is, in first approximation, independent of $\Phi$ and given by $\varepsilon_{\mathrm{Max}} \simeq 0.12 \omega$. Since $\varepsilon_{\mathrm{Max}} \ll \omega$, the energies of states in the first Landau level are well separated from the energies of states in the second Landau level and, therefore, 
the first Landau level retains its character*. By using the Stirling formula, the spectrum (2.22) for $L-\sqrt{L} \leq l \leq L+\sqrt{L}$ becomes

$$
\varepsilon_{l} \simeq \frac{v}{R}[(l-L)-\mu], \quad v=\sqrt{\frac{e B}{4 \pi m^{2}}},
$$

and we recognize $v$ and $\mu$ as the velocity and chemical potential parameters introduced in the previous section. In this context, the "light-velocity" $v$ has to be considered as a phenomenological parameter; its exact value depends on the real confining potential felt by the electrons and the value obtained in (2.24) has not to be taken as a prediction. The velocity will appear as an overall parameter in the emerging conformal field theory, whose universal results will be independent of its exact value. Note also that our approach gives a vanishing $\beta_{0}$ and is therefore appropriate to describe one edge of the annulus. The gaps for charged excitations on the disk geometry will be restored later in section 3 .

The crucial point in our treatment of the confining pressure is that the Hamiltonian $H_{R}$ in (2.20) automatically reduces to a $(1+1)$-dimensional Hamiltonian for the boundary $(r=R)$ values of the field operator (2.23). To see that these constitute the only degrees of freedom we employ an identity recently pointed out in ref. [28]:

$$
\left(D_{i} \Psi\right)^{\dagger}\left(D_{i} \Psi\right)=\left(D_{+} \Psi\right)^{\dagger}\left(D_{+} \Psi\right)+m \varepsilon^{i j} \partial_{i} J^{j}+e B \rho .
$$

Combining this with the self-duality condition (2.10) for $\Psi$ restricted to the first Landau level, we see that $H_{R}$ reduces to a pure boundary term given by

$$
H_{R}=\frac{1}{4 m} \int_{0}^{2 \pi R} d x \Psi^{\dagger}(-i)\left(\partial_{x}-i \frac{R}{\ell^{2}}\right) \Psi+\text { c.c. },
$$

where $x=R \theta$ is the 1-dimensional coordinate on the edge. Note that the Hamiltonian density of this "relativistic" $(1+1)$-dimensional theory is the transverse component of the non-relativistic current restricted to $r=R$.

We thus conclude that our modification of the original Hamiltonian to $H_{R}$ given in (2.20) corresponds to introducing a confining potential due to the background; the big advantage of our procedure is that it allows for an exact analytic treatment. As a bonus, we obtain an automatic reduction from a $(2+1)$-dimensional non-relativistic Hamiltonian to a $(1+1)$-dimensional "relativistic" Hamiltonian. This reduction is the microscopic analogue of the reduction in Chern-Simons gauge theories [29].

* For filling $\nu=1$ we can neglect the mixing $O\left(\exp \left(-(R / \ell)^{2}\right)\right)$ with higher Landau levels. 


\section{Thermodynamic limit and CFT on the cylinder}

\subsection{Thermodynamic limit}

We now study the thermodynamic limit $L=(R / \ell)^{2}-\mu \rightarrow \infty$ of the theory describing the dynamics at one edge of the Hall sample. In this limit, all quantities in the theory will acquire a manifest $(1+1)$-dimensional relativistic form and we shall find conformal invariance by explicit derivation of the Virasoro algebra. In eq.(2.26), we already derived the $(1+1)$-dimensional Hamiltonian. However, it is not obvious that the field operator $\Psi(r=R)$ reduces in the limit to the correct expansion for a relativistic theory. Nonetheless this is indeed the case, as we now discuss.

We first notice that the theory defined by the Hamiltonian (2.20) contains both an ultraviolet cutoff $\ell$ and an infrared cutoff $R$, which are tied together by the particle number $L \sim(R / \ell)^{2}$. The restriction to the linear range $|l-L|<\sqrt{L}$ of the spectrum $\varepsilon_{l}$ in eq.(2.24) amounts to the ultraviolet cut-off for the one-dimensional momentum $|p|=|(l-L) / R|<1 / \ell$, which becomes irrelevant in the limit $R \rightarrow \infty$. In this limit, the

Fock space of the operators $a_{l}, a_{l}^{\dagger}$, for angular momentum states can be identified with the Fock space of a relativistic $(1+1)$-dimensional fermion. Similarly, the filled Landau level becomes the Dirac sea for the relativistic fermion [9].

To study the behaviour of the field operator, it is convenient to shift variables as follows,

$$
\begin{aligned}
e_{l} & \equiv \varepsilon_{L+l}, \\
b_{l} & \equiv a_{L+l}, \\
\Psi(\mathbf{x}) & =\left(\frac{2}{\pi \ell^{2}}\right)^{1 / 4} e^{i(L+\mu) \theta} F_{R}^{\text {(cutoff) }}(\mathbf{x}) .
\end{aligned}
$$

When evaluated on the boundary, the field operator $F_{R}^{(\text {cutoff })}$ acquires the form

$$
\begin{aligned}
F_{R}^{(\text {cutoff })}\left(R e^{i \theta}, t\right) & =\sum_{l=-L}^{\infty} \frac{C_{l}}{\sqrt{2 \pi R}} e^{i(l-\mu) \theta} e^{-i e_{l} t}, \\
C_{l}^{2} & =\left.\frac{\sqrt{2 \pi}}{(L+l) !}\left(\frac{R}{\ell}\right)^{2 L+2 l+1} e^{-R^{2} / \ell^{2}}\right|_{R^{2}=(L+\mu) \ell^{2}} .
\end{aligned}
$$

In this expansion, the coefficients are approximated by $C_{l} \sim \exp \left(-(l-\mu)^{2} / 2 L\right)$ for $L \rightarrow \infty$. Since the width of this gaussian is $\sqrt{L}$, the $C_{l}$ give a smooth ultraviolet cutoff to the sum 
over $l$, which is limited to the range $|l|<\sqrt{L} \simeq R / \ell$ of linear energy (2.24). In this range, the wave functions have the correct $(1+1)$-dimensional form which ensures orthonormality in the Hilbert space on the circle. Note that to leading order for $L \rightarrow \infty$, we can remove this cutoff which would give subleading contributions $O(1 / \sqrt{L})=O(\ell / R)$. (This will be explicitly checked in section 4). Therefore, we obtain the approximate field operator

$$
F_{R}(\theta, t)=\sum_{l=-\infty}^{+\infty} \frac{1}{\sqrt{2 \pi R}} e^{i(l-\mu)\left(\theta-\frac{v}{R} t\right)} .
$$

In terms of the field $F_{R}$, the boundary Hamiltonian introduced in the previous section, eq.(2.26), takes the form

$$
H_{R}=\frac{v}{2} \int_{0}^{2 \pi R} d x F_{R}^{\dagger}\left(-i \partial_{x}\right) F_{R}+c . c ., \quad x=R \theta .
$$

Eqs. (3.3) and (3.4) are the field and the Hamiltonian of a relativistic chiral charged fermion* (Weyl fermion), moving with "light-velocity" $v$ on a circle of circumference $2 \pi R$.

A non-trivial aspect of the thermodynamic limit is the role of the chemical potential $\mu$. Eq.(3.3) allows a continuous spectrum of boundary conditions

$$
F_{R}(\theta+2 \pi)=e^{-i 2 \pi \mu} F_{R}(\theta)
$$

parametrized by the fractional part of $\mu$. Such general boundary conditions are possible for the Weyl fermion [18], and affect the spectrum of the theory, as we shall see later. The two cases mostly considered in string theory are called:

i) $\mu=0$ : Ramond (R), or periodic b.c. on the circle;

ii) $\mu=\frac{1}{2}$ : Neveu-Schwarz (NS), or anti-periodic b.c. on the circle.

\subsection{Conformal Field Theory on the Cylinder}

Conformal transformations in $(1+1)$ dimensions are analytic reparametrizations of one complex coordinate. A distinctive feature of a conformal invariant theory is the possibility to express all quantities as analytic functions of this complex coordinate, call it $\eta$ [15]. This variable parametrizes an Euclidean space-time plane. Our theory, given by eqs.(3.3) and (3.4) is defined on the Minkowskian cylinder $(R \theta, t)$ made by the circle and the time

* The chirality is determined by the sign of the external magnetic field $B$. 
coordinate. By rotating to Euclidean time $t=-i \tau$, we identify the conformal coordinate as

$$
\eta=e^{\frac{1}{R}(v \tau+i R \theta)}, \quad \operatorname{Arg} \eta=\operatorname{Arg} z,
$$

where $z$ parametrizes the original plane of the electrons. This mapping relates the cylinder with the conformal plane $\eta$, such that its unit circle $|\eta|=1$ corresponds to the edge $|z|=R$ (see Fig. 7).

The field operator (3.3) is anti-holomorphic in this variable,

$$
F_{R}(\bar{\eta})=\frac{\bar{\eta}^{\frac{1}{2}}}{\sqrt{2 \pi R}} \sum_{l=-\infty}^{+\infty} \bar{\eta}^{-l+\mu-\frac{1}{2}} b_{l}=\frac{\bar{\eta}^{\frac{1}{2}}}{\sqrt{2 \pi R}} F(\bar{\eta}) .
$$

Similarly, one finds

$$
F_{R}^{\dagger}(\bar{\eta})=\frac{\bar{\eta}^{\frac{1}{2}}}{\sqrt{2 \pi R}} \sum_{l=-\infty}^{+\infty} \bar{\eta}^{l-\mu-\frac{1}{2}} b_{l}^{\dagger}=\frac{\bar{\eta}^{\frac{1}{2}}}{\sqrt{2 \pi R}} F^{\dagger}(\bar{\eta})
$$

In these equations, we stripped off an additional term in order to make contact with the standard notation of CFT [18]. The equations of motion now coincide with the analyticity condition $\partial_{\eta} F=\partial_{\eta} F^{\dagger}=0$, and the Hamiltonian and $U(1)$ current densities are also chiral

$$
\begin{aligned}
\rho_{R}(\bar{\eta}) & =F_{R}^{\dagger} F_{R} \\
H_{R}=\frac{1}{i} \oint d \bar{\eta} \mathcal{H}_{R}(\bar{\eta}), \quad \mathcal{H}_{R}(\bar{\eta}) & =\frac{v}{2}\left(\partial_{\bar{\eta}} F_{R}^{\dagger} F_{R}-F_{R}^{\dagger} \partial_{\bar{\eta}} F_{R}\right) .
\end{aligned}
$$

The following table clarifies the meaning of these two densities in $(2+1)$ and $(1+1)$ dimensions.

$$
\begin{array}{crl}
\rho_{R} & \frac{\text { Non }- \text { rel. }(2+1)}{\text { charge density }} & \frac{\text { Rel. }(1+1)}{\text { chiral current }} \\
\mathcal{H}_{R} \quad \text { transverse current } & \text { stress tensor } \bar{T}(\bar{\eta})
\end{array}
$$

We now compute the chiral algebra of this field theory. To this end, we define the charges

$$
\begin{aligned}
\rho_{n} & \equiv \frac{R}{i} \oint d \bar{\eta} \rho_{R}(\bar{\eta}) \bar{\eta}^{n-1}, \\
L_{n} & \equiv \frac{R}{i v} \oint d \bar{\eta} \mathcal{H}_{R}(\bar{\eta}) \bar{\eta}^{n} .
\end{aligned}
$$

The charges $\rho_{n}$ and $L_{n}$ generate local gauge and conformal transformations. Global transformations are generated by $\rho_{0}$ and $\left\{L_{-1}, L_{0}, L_{1}\right\}$. Note that the total charge operator 
and the Hamiltonian of the $(1+1)$-dimensional relativistic theory are given by $\rho_{0}$ and $H_{R}=\frac{v}{R} L_{0}$, respectively. The dimensionless Hamiltonian $L_{0}$ of CFT will describe the $1 / R$ dependence of the spectrum of edge excitations. By using the field expansions (3.7) (3.8) in eq.(3.10), we obtain

$$
\begin{aligned}
\rho_{n} & =\sum_{l=-\infty}^{+\infty} b_{l-n}^{\dagger} b_{l} \\
L_{n} & =\sum_{l=-\infty}^{+\infty}\left(l-\frac{n}{2}-\mu\right) b_{l-n}^{\dagger} b_{l} .
\end{aligned}
$$

Clearly, $\rho_{0}$ and $L_{0}$ have to be regularized with some normal ordering prescription. To this end, we note that, with the notation introduced in (3.1), the original filled Landau level state $|\Omega\rangle$ is defined by

$$
\begin{aligned}
& b_{l}|\Omega\rangle=0, l>0, \\
& b_{l}^{\dagger}|\Omega\rangle=0, l \leq 0,
\end{aligned}
$$

and thus becomes the filled Dirac sea for the relativistic field theory of edge excitations. The normal ordering prescription for this ground state is obtained by writing the annihilation operators $b_{l}(l>0)$ and $b_{l}^{\dagger}(l \leq 0)$ to the right-hand side of the creation operators $b_{l}^{\dagger}$ $(l>0)$ and $b_{l}(l \leq 0)$. This prescription amounts to an infinite subtraction, therefore finite normal ordering constants should be included in $\rho_{0}$ and $L_{0}$, and they are fixed by algebraic conventions as follows.

The normal-ordered charges $\rho_{n}$ and $L_{n}$ satisfy the following chiral algebra

$$
\begin{aligned}
{\left[\rho_{n}, \rho_{m}\right] } & =n \delta_{n+m, 0} \\
{\left[L_{n}, \rho_{m}\right] } & =-m \rho_{n+m}, \\
{\left[L_{n}, L_{m}\right] } & =(n-m) L_{n+m}+\frac{c}{12}\left(n^{3}-n\right) \delta_{n+m, 0}, \quad c=1 .
\end{aligned}
$$

(The explicit computation is described for example in ref. [30]). The first algebra in (3.13) is the $U(1)$ Kac-Moody algebra for the generators $\rho_{n}$ of local gauge transformations; the third algebra is the Virasoro algebra for the generators $L_{n}$ of local conformal transformations. As is well known, the central extensions in these two algebras come from quantum effects associated with the infinite Dirac sea in the thermodynamic limit. The coefficient of $n^{3}$ in the Virasoro algebra does not depend on the finite normal-ordering constants and determines the central charge $c$. The latter is found to be $c=1$, as it should be for a Weyl fermion. Instead, the coefficient of the linear term in the Virasoro central extension can be shifted by redefinitions of $L_{0}$ by a constant. The convention in (3.13) is standard and 
fixes the value of $L_{0}|\Omega\rangle$. The value of $\rho_{0}|\Omega\rangle$ is fixed by the requirement of the absence of an anomaly in the mixed commutator. Altogether, the properties of the ground state are summarized by

$$
\begin{array}{llrl}
L_{n}|\Omega, \mu\rangle & =0, & \rho_{n}|\Omega, \mu\rangle & =0, n>0, \\
L_{0}|\Omega, \mu\rangle & =\left(\frac{1}{8}+\frac{\mu^{2}-\mu}{2}\right)|\Omega, \mu\rangle, & \rho_{0}|\Omega, \mu\rangle & =\left(\frac{1}{2}-\mu\right)|\Omega, \mu\rangle .
\end{array}
$$

Note the dependence of the ground state on the chemical potential $\mu$. In the thermodynamic limit, the filled Landau level is a highest-weight state of the $(c=1)$ chiral algebra (3.13) with charge $Q_{0}=\frac{1}{2}-\mu$ and conformal dimension $h_{0}=\frac{1}{8}+\frac{1}{2}\left(\mu^{2}-\mu\right)$. In the next section, we shall determine the value of $\mu$. Given that $|\Omega, \mu\rangle$ represents the original filled Landau level state, we recognize (3.14) as the algebraic characterization of the incompressibility of this state. It can be shown [31] that the highest-weight conditions (3.14) are equivalent to the $W_{\infty}$-symmetry conditions recently discussed in ref. [5].

Local conformal and gauge transformations, generated by the charges $L_{n}$ and $\rho_{m}$, are dynamical symmetries of the theory. This is reflected in the fact that the charges $L_{n}$ and $\rho_{m}$ do not commute with the Hamiltonian $L_{0}$; this non-commutativity is, however, compensated by the explicit time-dependence of the charges $L_{n}$ and $\rho_{m}$. Indeed, this is such that

$$
\begin{gathered}
\frac{d L_{n}}{d \tau}=\frac{\partial L_{n}}{\partial \tau}+\frac{v}{R}\left[L_{0}, L_{n}\right]=0, \\
\frac{d \rho_{n}}{d \tau}=\frac{\partial \rho_{n}}{\partial \tau}+\frac{v}{R}\left[L_{0}, \rho_{n}\right]=0 .
\end{gathered}
$$

This is sufficient to guarantee that all states in the theory fall into highest-weight representations of the chiral algebra (3.13), characterized by the eigenvalues $h$ of $L_{0}$ (conformal dimensions) and $Q$ of $\rho_{0}$ (charge). Such states are generated from the ground state by the action of primary fields $V_{h, Q}$ : these specify the operator content of the CFT [15] [18].

\subsection{Operator content of the CFT}

To extract the operator content of our theory, we compute the grand-canonical partition function on the torus obtained by compactifying the Euclidean time,

$$
\begin{aligned}
\mathcal{Z}(q, w) & \equiv \operatorname{Tr}\left(e^{-T H_{R}} w^{\rho_{0}}\right)=\operatorname{Tr}\left(q^{L_{0}-\frac{1}{24}} w^{\rho_{0}}\right) \\
q & \equiv \exp \left(-\frac{v T}{R}\right)
\end{aligned}
$$


where $T$ is the Euclidean time period and $w$ the fugacity. The factor $\frac{1}{24}$ in the exponent is the Casimir energy which will be discussed in detail in the next section. $\mathcal{Z}(q, w)$ is readily computed with standard statistical mechanics techniques, once we realize that our relativistic theory describes "electrons" with spectrum $(n-\mu)(n \geq 1)$, and "positrons" with spectrum $(n-(1-\mu))(n \geq 1)$, as is clear from (3.11):

$$
\mathcal{Z}(q, w)=q^{\frac{1}{12}+\frac{1}{2}\left(\mu^{2}-\mu\right)} w^{\frac{1}{2}-\mu} \prod_{n=1}^{\infty}\left(1+w q^{n-\mu}\right)\left(1+w^{-1} q^{n-(1-\mu)}\right)
$$

This expression can be further simplified by using the following version of the Jacobi triple product identity [27]:

$$
q^{\frac{1}{2}\left(\frac{1}{2}-\mu\right)^{2}} \prod_{n=1}^{\infty}\left(1-q^{n}\right)\left(1+w q^{n-\mu}\right)\left(1+w^{-1} q^{n-(1-\mu)}\right)=\sum_{n=-\infty}^{\infty} q^{\frac{1}{2}\left(n+\frac{1}{2}-\mu\right)^{2}} w^{n}
$$

Indeed, use of this identity leads to the final result:

$$
\mathcal{Z}(q, w)=\frac{1}{\eta(q)} \sum_{n=-\infty}^{\infty} q^{\frac{1}{2}\left(n+\frac{1}{2}-\mu\right)^{2}} w^{n+\frac{1}{2}-\mu}
$$

where $\eta(q)$ denotes the Dedekind funtion

$$
\eta(q)=q^{\frac{1}{24}} \prod_{n=1}^{\infty}\left(1-q^{n}\right)
$$

The partition function contains two basic factors. The factor $1 / \eta(q)$ represents the contribution from neutral particle-hole excitations across the Fermi level; the second factor is a sum over charged sectors which are in one-to-one correspondence with the primary fields $V_{h_{n}, Q_{n}}$. The exponents of $q$ and $w$ give their conformal dimensions $*$ and charges, respectively:

$$
\begin{aligned}
h_{n} & =\frac{1}{2}\left(n+\frac{1}{2}-\mu\right)^{2}, \\
Q_{n} & =n+\frac{1}{2}-\mu .
\end{aligned}
$$

Note that from the point of view of the CFT the integer part of $\mu$ is irrelevant since it can be reabsorbed by a shift of the summation index $n$ in eq.(3.19). Only the fractional

\footnotetext{
* Degenerate representations for $c=1$, having dimensions $h=k^{2} / 4, k \in \mathbf{Z}$, never arise here.
} 
part of $\mu$ is relevant, since it determines the boundary conditions. Actually, we are now going to argue that $\mu$ is dynamically fixed to be $1 / 2$.

To this end we first note that the confining potential and the non-degenerate spectrum $\varepsilon_{l}$ arise from the neutralizing background. Moreover, as we show in the next section, the renormalized charge operator $\rho_{0}$ is the physical charge, since it correctly couples to additional electro-magnetic fields, thereby leading to the measured Hall current. Therefore, the ground state should be a neutral eigenstate of $\rho_{0}$. We thus conclude that $\mu$ dynamically self-tunes to the value

$$
\mu=\frac{1}{2}
$$

This implies that the ground state $|\Omega\rangle \equiv\left|\Omega, \mu=\frac{1}{2}\right\rangle$ is $S L(2, \mathbf{C})$ invariant, since it is annihilated by all three generators of global conformal trasformations, $L_{k}|\Omega\rangle=0, k=$ $-1,0,1$. It implies also the absence of spin fields 32 from the spectrum of the CFT.

With $\mu=1 / 2$, the conformal dimensions and charges of the primary fields reduce to

$$
\begin{aligned}
h_{n} & =\frac{1}{2} n^{2}, \\
Q_{n} & =n .
\end{aligned}
$$

Every one of these primary fields can be obtained by operator product expansion of the two basic fields $V_{\frac{1}{2},-1} \equiv F$ and $V_{\frac{1}{2},+1} \equiv F^{\dagger}$, where $F, F^{\dagger}$ are the fermion fields previously introduced in eqs. (3.7), (3.8). We thus conclude that the theory describing the dynamics of the edge excitations in the thermodynamic limit is the $c=1$ CFT of a Weyl fermion with Neveu-Schwarz boundary conditions.

The Hall system on the annulus geometry can be described by taking a copy of the CFT for each boundary. The one-particle spectrum for the annulus of radii $R_{1}, R_{2}$ described in section 2 (see Fig. 4) can be reproduced in our approach by the Hamiltonian

$$
H_{R_{1}, R_{2}}=\frac{1}{2 m} \int_{R_{1}<r<R_{2}} d^{2} \mathbf{x}\left(\left(D_{i} \Psi\right)^{\dagger}\left(D_{i} \Psi\right)-B \rho\right)=H_{R_{2}}-H_{R_{1}},
$$

where $H_{R_{1}}$ and $H_{R_{2}}$ are two copies of the previously discussed Hamiltonian. For $R_{2}-R_{1} \rightarrow \infty$, the one-dimensional Hilbert spaces at each edge are coupled only by overall charge conservation. The total partition function is thus given by

$$
\begin{aligned}
\mathcal{Z}_{\text {annulus }} & =\frac{1}{\eta(q) \eta(\bar{q})} \sum_{n=-\infty}^{+\infty}(q \bar{q})^{\frac{1}{2} n^{2}} w^{n}, \\
q & \equiv \exp \left(-\frac{v_{2} T}{R_{2}}\right), \quad \bar{q} \equiv \exp \left(-\frac{v_{1} T}{R_{1}}\right) .
\end{aligned}
$$


While neutral excitations at the two edges remain independent, charged excitations require the transfer of particles from one edge to the other.

\subsection{Deformed CFT on the disk geometry}

As emphasized in section 2, on a disk geometry the charged excitations acquire a gap that spoils conformal symmetry. Nonetheless, much information can be extracted from the CFT derived in the preceding sections. The crucial point is that $\rho_{0}$ is a Casimir of the chiral algebra (3.13). Therefore one can modify the Hamiltonian by adding to it any function of $\rho_{0}$ without changing the Hilbert space of the theory. We can add the gaps by hand with the following redefinition:

$$
H_{R} \rightarrow \hat{H}_{R}=\frac{v}{R} L_{0}+\gamma\left(\rho_{0}\right), \quad \gamma(n)= \begin{cases}n \beta_{0}, & n \geq 0 \\ n\left(\Delta-\beta_{0}\right), & n<0\end{cases}
$$

In any irreducible representation of the chiral algebra with charge $n$ the additional piece is a multiple of the identity. The infinite charges $\rho_{n}$ and $L_{n}$ are still (dynamically) conserved with respect to the modified Hamiltonian $\hat{H}_{R}$ as in eq.(3.15). Therefore it still makes sense to label states of the Hilbert space according to the eigenvalues of these charges. The resulting deformed $\mathrm{CFT}$ is fully consistent. Its partition function is given by

$$
\mathcal{Z}_{\text {disk }}=\frac{1}{\eta(q)} \sum_{n=-\infty}^{+\infty} q^{\frac{1}{2} n^{2}+\frac{R}{v} \gamma(n)} w^{n}
$$

The "conformal part" $v L_{0} / R$ of the Hamiltonian $\hat{H}_{R}$ determines the universal $1 / R$ corrections to the non-universal gaps $\beta_{0}$ and $\left(\Delta-\beta_{0}\right)$.

Note that the presence of gaps on the disk geometry has been somewhat neglected in the literature on edge excitations. In particular, the effective Chern-Simons theory approach applies, strictly speaking, only to fully gapless geometries like the annulus. 


\section{Integer Quantum Hall Effect: the CFT point of view}

In this section, we shall explore the implications of the CFT derived in the previous section for the physics of the $\nu=1$ quantum Hall effect. Clearly, this effective theory describes only some properties of the quantum Hall states; however, conformal invariance implies that these properties are universal.

\subsection{Finite-size spectra on the disk geometry}

In section 3 we have explained how all quantities of the CFT on the cylinder can be obtained from the corresponding quantities on the plane $\eta$ by the conformal map (3.6). In the case of the Hamiltonian, however, we did not pay attention to normal ordering effects in eq.(3.10). The correct Hamiltonian [20] on the cylinder is given by

$$
H_{R}=\frac{v}{R}\left(L_{0}-\frac{c}{24}\right), \quad(c=1)
$$

The additional term depends on the central charge of the theory and is called the Casimir energy. It arises from the mismatch of normal ordering procedures on the plane and on the cylinder.

Suppose we have computed the ground-state energy of the $\nu=1$ quantum Hall state in the disk geometry of radius $R$. The finite-size expansion of this energy will be generically

$$
E_{0}=\frac{R^{2}}{\ell^{3}} \alpha_{2}+\frac{R}{\ell^{2}} \alpha_{1}+\frac{1}{\ell} \alpha_{0}+\frac{1}{R} \alpha_{-1}+\frac{\ell}{R^{2}} \alpha_{-2}+\ldots
$$

In this equation, $\ell$ is the magnetic length (the ultraviolet cutoff) and $\alpha_{n}$ are dimensionless coefficients that can depend on $\ell$, the electron mass $m$ and other parameters of the microscopic Hamiltonian. The leading term gives a finite energy per particle since $(R / \ell)^{2}$ is the number of electrons. The other terms are subleading in the experimental regime of large flux through the sample, $\Phi=(R / \ell)^{2} \gg 1$. On the other hand, the result of the CFT is given by eq.(4.1) combined with $L_{0}|\Omega\rangle=0$ :

$$
E_{0}=\left\langle\Omega\left|H_{R}\right| \Omega\right\rangle=-\frac{v}{24} \frac{1}{R}
$$

Actually, in the CFT the terms $\alpha_{2}, \alpha_{1}$ and $\alpha_{0}$ have been put to zero by the infinite subtraction of normal ordering. The terms $\alpha_{-2}, \alpha_{-3}, \ldots$ represent the higher order corrections for $R \rightarrow \infty$ neglected in the thermodynamic limit of section 3 . Therefore, CFT leads to the 
prediction $\alpha_{-1}=-\frac{v}{24}$. The discussion of section 3 shows that this prediction is universal because it is independent of the details of the confining potential. The velocity $v$ is a free parameter; it can be determined from the spectrum of excitations as we discuss hereafter. Note that the CFT prediction is a subleading correction to the bulk properties discussed by Laughlin 12 and verified in boundaryless geometries [33.

The Casimir energy (4.1) implies a universal term

$$
P=-\frac{v}{48 \pi} \frac{1}{R^{3}}
$$

in the pressure $P$ of the electron liquid. An independent measurement of the electron liquid pressure and the effective velocity $v$ of edge excitations, might thus provide an experimental verification of the CFT. Another possibility is the measurement of the contribution of the Casimir energy to the specific heat [8].

In order to illustrate the finite-size expansion (4.2), let us compute the ground state energy $E_{0}$ directly by using the spectrum (2.22). This gives

$$
E_{0}=\sum_{l=0}^{L} \frac{\omega}{2} \frac{\Phi^{l} e^{-\Phi}}{l !}(l-\Phi)=-\frac{\omega}{2} e^{-\Phi} \frac{\Phi^{L+1}}{L !} .
$$

Using $\Phi=(R / \ell)^{2}=L+\frac{1}{2} \quad\left(\mu=\frac{1}{2}\right)$, and Stirling's formula

$$
L !=L^{L} e^{-L} \sqrt{2 \pi L}\left(1+\frac{1}{12 L}+O\left(\frac{1}{L^{2}}\right)\right),
$$

we obtain the expansion of $E_{0}$ :

$$
E_{0}=-v \frac{R}{\ell^{2}}-\frac{v}{24} \frac{1}{R}+O\left(\frac{\ell}{R^{2}}\right) .
$$

As expected, the $1 / R$-term is in perfect agreement with the prediction of the CFT.

A further result of the CFT is that excitations about the incompressible filled Landau level state can all be classified according to representations of the chiral algebra (3.13). As we discussed in section 3, there are two types of such excitations: neutral particle-hole excitations (edge excitations), and charged excitations. The charged excitations are in one to one correspondence with the primary fields of the chiral algebra, and are identified with the quasi-hole and quasi-particle excitations of Laughlin's theory. The spectrum of excitations can be extracted from the partition function (3.27) :

$$
\Delta E_{n, k} \equiv E_{n, k}-E_{0}=\gamma(k)+\frac{v h_{k}}{R}+\frac{v n}{R},
$$


with $n, k \in \mathbf{Z}$ and $0<n \ll \sqrt{L}$. The second term is the universal $1 / R$-correction to the gap and is determined by the conformal dimension $h_{k}=k^{2} / 2$ of the primary fields. Conformal invariance implies that this is the same for quasi-particles and quasi-holes. The third term is the spectrum of neutral edge excitations in any given charged sector labelled by $k$ (the conformal family).

Numerical simulations in the disk geometry were carried out in refs. [34] [35] [11]. The authors of refs. 350 11] concentrated on neutral edge excitations in systems of interacting electrons. These studies show a good agreement with the multiplicities predicted by conformal invariance (the $1 / \eta$-term in the partition function (3.27), but did not yet verify the linear relativistic spectrum. Finite-size effects in the spectrum were addressed in ref. [34]; unfortunately, the numerical precision is not yet sufficient to allow a quantitative comparison with our predictions. It would be most interesting to improve these analyses.

\subsection{Monodromies and statistics of excitations}

An important consequence of CFT is that it predicts the monodromies of the primary fields. Through analytic continuation, these determine then the statistics of the quasi-particle and quasi-hole excitations. The identification between CFT correlators and physical wave functions is done at $t=0$, and involves therefore only static properties of the CFT. The deformation of the CFT discussed in the previous section is not relevant for the following discussion of monodromies.

Let us compute the conformal correlators of two primary fields at points $\bar{\eta}_{1}$ and $\bar{\eta}_{2}$ :

$$
\begin{aligned}
\left\langle\Omega\left|b_{0}^{\dagger} b_{-1}^{\dagger} F\left(\bar{\eta}_{1}\right) F\left(\bar{\eta}_{2}\right)\right| \Omega\right\rangle & =\left(\bar{\eta}_{1}-\bar{\eta}_{2}\right), \\
\left\langle\Omega\left|b_{1} b_{2} F^{\dagger}\left(\bar{\eta}_{1}\right) F^{\dagger}\left(\bar{\eta}_{2}\right)\right| \Omega\right\rangle & =\left(\bar{\eta}_{1}-\bar{\eta}_{2}\right) .
\end{aligned}
$$

Note that charge conservation requires the ground state of charge $\mp 2$ on the left-hand side of these expressions. By applying the conformal map (3.6), and restoring the factors extracted in (3.1) (3.7) (3.8), we obtain the wave-functions of the corresponding quasi-hole and quasi-particle excitations, located in the physical plane at $z_{1}=R e^{i \theta_{1}}$ and $z_{2}=R e^{i \theta_{2}}$ :

$$
\begin{aligned}
\left\langle\Omega\left|b_{L}^{\dagger} b_{L-1}^{\dagger} \Psi\left(R e^{i \theta_{1}}\right) \Psi\left(R e^{i \theta_{2}}\right)\right| \Omega\right\rangle & =\frac{1}{\sqrt{2 \pi^{3}} R \ell} e^{i(L-1)\left(\theta_{1}+\theta_{2}\right)}\left(e^{i \theta_{2}}-e^{i \theta_{1}}\right), \\
\left\langle\Omega\left|b_{L+1} b_{L+2} \Psi^{\dagger}\left(R e^{i \theta_{1}}\right) \Psi^{\dagger}\left(R e^{i \theta_{2}}\right)\right| \Omega\right\rangle & =\frac{1}{\sqrt{2 \pi^{3}} R \ell} e^{-i(L+1)\left(\theta_{1}+\theta_{2}\right)}\left(e^{-i \theta_{1}}-e^{-i \theta_{2}}\right) .
\end{aligned}
$$


It is easy to check that this is exactly the result one would get by direct computation of the same quantities using the original field operator of the Landau levels. In particular, the monodromies (4.9) determine the analytic parts $\left(z_{1} z_{2}\right)^{L-1}\left(z_{2}-z_{1}\right)$ and $\left(\bar{z}_{1} \bar{z}_{2}\right)^{L+1}\left(\bar{z}_{1}-\bar{z}_{2}\right)$ of the quasi-hole and quasi-particle wave-functions and, therefore, also the fermionic statistics of these excitations. The fact that we are dealing with a $c=1 \mathrm{CFT}$ guarantees the polynomial character of multi-excitations wave-functions; this ensures in turn that these excitations carry Abelian statistics.

The above computation clarifies the formal analogy between conformal correlators and Laughlin's wave-functions pointed out by several authors [21] 22] 223] 24]. The conformal correlators are defined only for $r=R$. However, by analytic continuation, they determine the analytic part of the excitation wave-functions of the full $(2+1)$-dimensional nonrelativistic theory. Therefore, the correlators of the CFT are indeed sufficient to fix the statistics of the excitations. However, we stress that the main relation is between conformal correlators and excitation wave-functions, not Laughlin's ground-state wave-functions.

\subsection{Long-range order on the boundary}

Conformal invariance dictates the form of the two-point function of primary fields of conformal dimension $h$. This has the power-law behaviour

$$
\left\langle\Omega\left|F^{\dagger}\left(\bar{\eta}_{1}\right) F\left(\bar{\eta}_{2}\right)\right| \Omega\right\rangle=\frac{1}{\left(\bar{\eta}_{1}-\bar{\eta}_{2}\right)^{2 h}}, \quad h=\frac{1}{2} .
$$

We can easily obtain the corresponding two-point function of the CFT on the cylinder by applying the conformal map (3.6) and restoring factors as in (4.10). We thus obtain the density matrix for points on the boundary:

$$
\left\langle\Omega\left|\Psi^{\dagger}\left(R e^{i \theta_{1}}\right) \Psi\left(R e^{i \theta_{2}}\right)\right| \Omega\right\rangle=\sqrt{\frac{2}{\pi \ell^{2}}} \frac{1}{2 \pi R} e^{i L\left(\theta_{2}-\theta_{1}\right)} \frac{e^{i \theta_{2}}}{e^{i \theta_{2}}-e^{i \theta_{1}}} .
$$

This implies that the filled Landau level state $|\Omega\rangle$ supports algebraic long-range order on the boundary, i.e. the absolute value of the density-matrix for points on the boundary at $r=R$ is proportional to the inverse of their distance (in the $(2+1)$-dimensional sense). This long-range order is characterized by the exponent $2 h=1, h$ being the conformal dimension of the primary fields entering the density-matrix. In Appendix A, we check that this non-trivial result can be also obtained directly from the Landau level expression of 
the density matrix by a rather involved computation. This confirms further the validity of the predictions of the CFT.

\subsection{Hall current in the annulus geometry}

We now derive the Hall current from the $(1+1)$-dimensional point of view. To this end we consider the theory at one edge of the annulus, say the outer one, and we add a flux tube at the center:

$$
\delta B=2 \pi \Phi_{0} \delta^{(2)}(\mathbf{x})
$$

In our circular geometry, the appropriate choice of gauge to describe this additional magnetic field is

$$
\delta A_{i}=\Phi_{0} \epsilon^{i j} \frac{x^{j}}{|\mathbf{x}|^{2}} .
$$

Accordingly, the one-particle Hamiltonian (2.1) is modified to

$$
H=-\frac{1}{2 m}(\nabla-i e(\mathbf{A}+\delta \mathbf{A}))^{2}-\frac{e}{2 m}(B+\delta B)
$$

The normalized energy and angular momentum eigenstates of this new problem are

$$
\hat{\psi}_{n, l}=e^{i e \Phi_{0} \theta} \psi_{n, l}
$$

where $\psi_{n, l}$ are the Landau level wave-functions (2.5). While the energy eigenvalues $\omega_{n}$ corresponding to $\hat{\psi}_{n, l}$ are unchanged, the angular momentum eigenvalues are modified to $\left(l+e \Phi_{0}\right)$. Following all the steps that led to (3.3) and (3.4), we find the following modifications due to the additional flux tube $\delta B$ :

$$
\begin{aligned}
H_{R} & =\frac{v}{2} \int_{0}^{2 \pi R} d x \hat{F}_{R}^{\dagger}\left(-i \partial_{x}+e \delta A_{x}\right) \hat{F}_{R}+c . c . \\
\delta A_{x} & =-\frac{\Phi_{0}}{R} \quad, \quad \hat{F}_{R}=e^{i e \frac{\Phi_{0}}{R} x} F_{R} .
\end{aligned}
$$

We now allow $\Phi_{0}$ to vary in time; this corresponds to a transverse electric field $E^{i} \equiv \partial_{t} A_{i}=\dot{\Phi}_{0} \epsilon^{i j} x^{j}|\mathbf{x}|^{2}$, which reduces to a $(1+1)$-dimensional uniform electric field

$$
E=\partial_{t} \delta A_{x}=-\frac{\dot{\Phi}_{0}}{R},
$$

on the boundary at $r=R$. The effective theory (4.17) describes therefore a $(1+1)$ dimensional Weyl fermion coupled to an external electric field. As it is well-known, such 
a theory is affected by the chiral anomaly [36], which amounts to a violation of charge conservation due to the external electric field. In the formulation (4.17), the origin of this phenomenon is hidden in the time-dependence of the normal ordering procedure. However, the anomaly can be exposed by performing a time-dependent transformation to the $\delta A_{x}=0$ gauge. In this gauge, we recover the previous field operator $F_{R}$; the price for this is, however, an additional piece proportional to the density in the Hamiltonian:

$$
\begin{aligned}
H_{R} & =\frac{v}{2} \int_{0}^{2 \pi R} d x F_{R}^{\dagger}\left(-i \partial_{x}\right) F_{R}+c . c .+\int_{0}^{2 \pi R} d x e \delta A_{0} \rho_{R} \\
\delta A_{0} & =\frac{\dot{\Phi}_{0}}{R} x \quad, \quad E=-\partial_{x} A_{0} .
\end{aligned}
$$

We can now compute the time-variation of the normal-ordered density $\rho_{R}$ by simply commuting it with the Hamiltonian $H_{R}$. The commutator of the first term in $H_{R}$ with $\rho_{R}$ is fixed by the second algebra in (3.13) and gives $\left(-v \partial_{x} \rho_{R}\right)$; the commutator of the additional term in $H_{R}$ with $\rho_{R}$ is determined by the first algebra in (3.13). It is non-vanishing due to the central extension in this Kac-Moody algebra and leads to the anomaly equation

$$
\left(\partial_{t}+v \partial_{x}\right) \rho_{R}=\frac{e}{2 \pi} E
$$

This equation tells us the rate of production of particles by the external electric field: $\partial_{t} Q=e E$. From the $(1+1)$-dimensional point of view, this charge pops out from the Dirac sea (spectral flow picture), which is an infinite reservoir. The identification of the Dirac sea with the quantum Hall droplet allows then to interpret the anomaly as a radial flow of charge through the boundary at $R$, i.e., a Hall current

$$
J^{i}=\frac{e}{2 \pi} \epsilon^{i j} E^{j}
$$

Note however that in the $(2+1)$-dimensional non-relativistic theory there is no net anomaly. The charge flowing through the outer boundary is provided by the inner boundary, and thus the anomalies of the CFTs at the two edges cancel each other. 


\section{Chiral boson and its canonical quantization}

It is well known that a Weyl fermion is equivalent to a free chiral boson [32]. The free fermion and the free boson give two equivalent representations of the chiral algebra discussed above. However, the free boson theory is more convenient because it can also represent other $c=1 \mathrm{CFTs}$, which correspond to interacting fermions. In the next section, we shall apply these to the fractional Hall effect.

There are two basic approaches to studying chiral $c=1$ CFTs. One is based on the field operator algebra and conformal representation theory; the other is canonical quantization based on an action. In the first approach, it is consistent to consider one chiral sector of the usual Klein-Gordon field, and to construct a set of primary fields which is closed under operator product expansion [18]. There are many consistent sets of fields with both integer and fractional conformal dimensions.

As it will become clear in the following, we need an action formulation in order to fix the field content for fractional Hall states. Previous canonical approaches turn out to be insufficient for our purposes. The Siegel action, quantized in ref. [37], describes CFTs with integer dimensions only. A better candidate is the first-order action of Floreanini and Jackiw [19]. By quantizing it on a compact space, and by introducing and additional coupling constant $\kappa$, we shall obtain sets of fields with fractional dimensions appropriate for the fractional Hall effect.

Consider a real boson $\phi(x, t)$, living on a circle of length $2 \pi R$, and with dynamics governed by the action

$$
S=-\frac{\kappa}{4 \pi} \int_{-\infty}^{+\infty} d t \int_{0}^{2 \pi R} d x\left(\partial_{t}+v \partial_{x}\right) \phi \partial_{x} \phi
$$

where $v$ is the "light-velocity". By rescaling the space-time variables as $x \rightarrow R \theta, t \rightarrow R t$, we can write the corresponding Hamiltonian in the form

$$
\begin{aligned}
H_{R} & =\frac{v}{R} L_{0}, \\
L_{0} & =\frac{\kappa}{4 \pi} \int_{0}^{2 \pi} d \theta\left(\partial_{\theta} \phi\right)^{2},
\end{aligned}
$$

where $L_{0}$ is again the dimensionless Hamiltonian of CFT.

Since the theory is defined on a compact space, special care is needed in treating boundary conditions. We impose boundary conditions

$$
\phi(2 \pi, t)-\phi(0, t)=-2 \pi \alpha_{0},
$$


parametrized by a time-independent constant $\alpha_{0}$. As a consequence, the real boson $\phi$ has to be considered as an angular variable, compactified on a circle, and $\alpha_{0}$ becomes correspondingly quantized, as we discuss below. By considering variations that preserve (5.3), we find that the action (5.1) is minimized by fields that satisfy the equation of motion

$$
\left(\partial_{t}+v \partial_{\theta}\right) \partial_{\theta} \phi=0
$$

with boundary conditions

$$
\begin{aligned}
\partial_{\theta} \phi(2 \pi, t) & =\partial_{\theta} \phi(0, t) \\
\left(\partial_{t}+v \partial_{\theta}\right) \phi(2 \pi, t) & =\left(\partial_{t}+v \partial_{\theta}\right) \phi(0, t) .
\end{aligned}
$$

The general solution of the equation of motion (5.4) can be written as

$$
\phi(\theta, t)=f(\theta-v t)+g(t)
$$

Here, $g(t)$ represents the gauge degree of freedom corresponding to the invariance of the action (5.1) and the Hamiltonian (5.2) under $\phi \rightarrow \phi+\lambda(t)$. Since it does not represent a physical degree of freedom, we discard it by imposing the gauge condition

$$
\left(\partial_{t}+v \partial_{\theta}\right) \phi=0
$$

In this gauge, the general solution of (5.4) compatible with the boundary conditions (5.3) and $(5.5)$, is given by

$$
\begin{aligned}
\phi(\theta-v t) & =\phi_{0}-\alpha_{0}(\theta-v t)+i \sum_{n \neq 0} \frac{\alpha_{n}}{n} \mathrm{e}^{i n(\theta-v t)}, \\
\alpha_{n}^{*} & =\alpha_{-n} .
\end{aligned}
$$

Upon quantization, the coefficients $\phi_{0}, \alpha_{0}$ and $\alpha_{n}$ of this expansion become operators acting on a bosonic Fock space, whose vacuum $|\Omega\rangle$ is defined by

$$
\alpha_{n}|\Omega\rangle=0, n>0
$$

The commutation relations of these operators are inferred from the corresponding commutation relations of the field $\phi$ as follows. The action (5.1) is of first order in time derivatives, and, therefore, the canonical momentum is completely specified in terms of the field $\phi$ and its space derivative. Nonetheless, there is no need to resort to the Dirac formalism to quantize the theory, as was recently emphasized by Faddeev and Jackiw [38]. 
The canonical commutation relations can be simply read off from the symplectic structure evident in the action (5.1):

$$
\left[\phi(\theta, t), \phi\left(\theta^{\prime}, t\right)\right]=i \frac{\pi}{\kappa} \varepsilon\left(\theta-\theta^{\prime}\right)
$$

These imply the commutation relations

$$
\begin{aligned}
{\left[\alpha_{0}, \phi_{0}\right] } & =\frac{1}{i \kappa}, \\
{\left[\alpha_{n}, \alpha_{m}\right] } & =\frac{n}{\kappa} \delta_{n+m, 0},
\end{aligned}
$$

for the coefficients of the expansion (5.8). In terms of these operators, the Hamiltonian (5.2) takes the form

$$
L_{0}=\frac{\kappa}{2} \alpha_{0}^{2}+\kappa \sum_{n=1}^{\infty} \alpha_{-n} \alpha_{n} .
$$

Note that this expression has already been normal ordered, by writing all the Fock space annihilators to the right. In analogy with (3.10), we also define the charges $L_{n}$, for $n \neq 0$, by taking moments of the Hamiltonian density (5.2) :

$$
L_{n} \equiv \frac{\kappa}{4 \pi} \int_{0}^{2 \pi} d \theta\left(\partial_{\theta} \phi\right)^{2} e^{-i n(\theta-v t)}=\frac{\kappa}{2} \sum_{l=-\infty}^{+\infty} \alpha_{n-l} \alpha_{l}
$$

By using the commutation relations (5.11), we finally obtain the chiral algebra of the bosonic theory:

$$
\begin{aligned}
{\left[\alpha_{n}, \alpha_{m}\right] } & =\frac{n}{\kappa} \delta_{n+m, 0} \\
{\left[L_{n}, \alpha_{m}\right] } & =-m \alpha_{n+m} \\
{\left[L_{n}, L_{m}\right] } & =(n-m) L_{n+m}+\frac{1}{12}\left(n^{3}-n\right) \delta_{n+m, 0} .
\end{aligned}
$$

This shows that the central charge is $c=1$.

In the following, we show that consistency of quantization forces $\kappa$ to be rational. Let us write $\kappa=p / q$, with $p$ and $q$ coprimes integers, and choose $p>q$ without loss of generality. From the boundary condition (5.3), $\phi(\theta)$ is a topologically non-trivial mapping of the circle to itself. We then choose the compactification radius of $\phi$ as $2 \pi / p$,

$$
\phi(\theta, t) \equiv \phi(\theta, t)+\frac{2 \pi}{p}
$$


which leads to the quantization condition

$$
\alpha_{0}=\frac{n}{p}, n \in \mathbf{Z}
$$

Since $\kappa \alpha_{0}$ is the canonical momentum conjugate to $\phi_{0}$, it follows that $\phi_{0}$ is an angular variable of range $[0,2 \pi q]$. This is also consistent with $\phi(\theta, t)$ being an angular variable of range $\left[0, \frac{2 \pi}{p}\right]$, due to the rationality of $\kappa$.

We are now ready to discuss the operator content of the bosonic theory. We first identify $\alpha_{0}$ with the total electromagnetic charge (this identification will be justified in detail below). Then we compute the grand-canonical partition function

$$
\mathcal{Z}(q, w)=\operatorname{Tr} q^{L_{0}-\frac{1}{24}} w^{\alpha_{0}}
$$

with $q$ and $w$ defined as in (3.16). Standard statistical mechanics techniques give

$$
\mathcal{Z}(q, w)=\frac{1}{\eta(q)} \sum_{n=-\infty}^{\infty} q^{\frac{1}{2} \frac{n^{2}}{p q}} w^{\frac{n}{p}}
$$

where $\eta(q)$ is the Dedekind function (3.20) and we have used the quantization condition (5.16). For $p q \neq 2 N^{2}$, the conformal dimensions* and charges of the primary fields are thus

$$
\begin{aligned}
h_{n} & =\frac{n^{2}}{2 p q}, \\
Q_{n} & =\frac{n}{p} .
\end{aligned}
$$

The primary fields are the Fubini-Veneziano vertex operators

$$
V_{\beta}(\bar{\eta})=: e^{i \beta \phi(\bar{\eta})}:,
$$

where $\bar{\eta}$ is the conformal variable in eq.(3.6). These satisfy

$$
\begin{aligned}
& {\left[L_{0}, V_{\beta}\right]=\left(\bar{\eta} \frac{\partial}{\partial \bar{\eta}}+\frac{\beta^{2}}{2 \kappa}\right) V_{\beta},} \\
& {\left[\alpha_{0}, V_{\beta}\right]=\frac{\beta}{\kappa} V_{\beta},}
\end{aligned}
$$

confirming that $V_{\beta}$ is a primary field of conformal dimension $h_{\beta}=\beta^{2} / 2 \kappa$ and charge $Q_{\beta}=\beta / \kappa$. To obtain the primary fields of our theory, we have, therefore, to restrict $\beta$ to

* For $p q=2 N^{2}$ we face the problem of degenerate representations of the Virasoro algebra, and more care is required in extracting the conformal dimensions of the primary fields [18]. 
the values $\beta_{n}=n / q$. For later convenience, we recall the operator product expansion of vertex operators:

$$
V_{\beta_{1}}\left(\bar{\eta}_{1}\right) V_{\beta_{2}}\left(\bar{\eta}_{2}\right)=\left(\bar{\eta}_{1}-\bar{\eta}_{2}\right)^{\beta_{1} \beta_{2} / \kappa}: V_{\beta_{1}}\left(\bar{\eta}_{1}\right) V_{\beta_{2}}\left(\bar{\eta}_{2}\right):
$$

We now discuss the special case $\kappa=1$. For $\kappa=1$, the operator content (5.19) of the bosonic theory coincides with the operator content (3.23) of the fermionic theory which describes edge excitations. In particular, the fermion fields are expressed by the bosonic vertex operators $V_{ \pm 1}$, since both are the primary fields of conformal dimension $1 / 2$ and charge \pm 1 in the respective representations: $F=V_{-1}, F^{\dagger}=V_{+1}$. By using (5.22), one actually verifies the Minkowskian anticommutators

$$
\begin{aligned}
& \left\{V_{ \pm 1}\left(\theta_{1}-v t\right), V_{ \pm 1}\left(\theta_{2}-v t\right)\right\}=0 \\
& \left\{V_{-1}\left(\theta_{1}-v t\right), V_{+1}\left(\theta_{2}-v t\right)\right\}=2 \pi \delta\left(\theta_{1}-\theta_{2}\right)
\end{aligned}
$$

The charge density on the cylinder $\rho_{R}=: F_{R}^{\dagger} F_{R}$ : can be accordingly expressed in terms of the bosonic field. Normal ordering can be implemented by subtracting the short-distance divergent part in eq.(5.22), thereby obtaining

$$
\rho_{R}=-\frac{1}{2 \pi} \partial_{x} \phi
$$

which, in turn, implies the mapping $\rho_{n}=\alpha_{n}$. In particular, the total charge $\rho_{0}$ is represented in the bosonic theory by $\alpha_{0}$, which justifies the previous identification.

Having identified $-\frac{1}{2 \pi} \partial_{x} \phi$ with the fermionic charge density $\rho_{R}$ on the cylinder it is easy to couple the chiral boson to $U(1)$ gauge fields. This is accomplished by adding to the action (5.1) the additional piece

$$
\Delta S=\frac{e}{2 \pi} \int_{-\infty}^{+\infty} d t \int_{0}^{2 \pi R} d x\left(A_{t} \partial_{x} \phi-A_{x} \partial_{t} \phi\right) .
$$

This coupling is Lorentz and gauge invariant (note that only $A_{\mu}$ 's are changed by gauge transformations). Since the fermion current density is also $\rho_{R}$, due to the chiral nature of the theory, eq.(5.25) reduces to the standard coupling $-A_{\mu} J^{\mu}$ in the physical gauge (5.7). The equation of motion is changed to

$$
\left(\partial_{t}+v \partial_{x}\right)\left(-\frac{1}{2 \pi} \partial_{x} \phi\right)=\frac{e}{2 \pi \kappa} E,
$$

with $E=\partial_{t} A_{x}-\partial_{x} A_{t}$ the electric field. For $\kappa=1$ it reduces exactly to the chiral anomaly (4.20) of the fermionic representation.

We thus conclude that the $c=1$ CFT describing the edge dynamics of one completely filled Landau level admits a bosonic representation. This can be easily generalized by varying the rational parameter $\kappa$. 


\section{Fractional quantum Hall effect}

In this section, we address the physics of a fractionally filled Landau level. Our approach is as follows. We suppose an incompressible ground state is formed at a rational value $\nu<1$. Specifically, we consider the Laughlin states at $\nu=1 / m, m$ odd integer. In these cases there exist short-range interactions such that these states are the exact incompressible ground states [2]. Next, we assume that the universal long-range properties of these states can be described by a $c=1$ CFT of interacting fermions, or equivalently, by the chiral boson theory of the previous section. Presently, we are not able to derive directly this CFT by applying the limit on the boundary of section 3. Nevertheless, some symmetry arguments support our assumption.

First, we can argue on general grounds that incompressibility in the bulk should amount to conformal invariance on the boundary, i.e. the ground state satisfies the highestweight conditions (3.14) $(\mu=1 / 2)$. Indeed, in the case $\nu=1$ these conditions are equivalent [31] to the area-preserving ( $W_{\infty}$-symmetry) conditions of the bulk theory studied in ref. [5]. There we showed that the $\nu=1 / m$ Laughlin ground states also possess the same symmetry; therefore we can expect again conformal invariance on the boundary. Secondly, we expect that the central charge is again $c=1$. Qualitatively, we know that the neutral excitations at $\nu=1 / m$ with short-range potentials are the same as in the $\nu=1$ theory. Following Laughlin, we only expect a modification of the charged excitations, which should have Abelian statistics.

Actually, Laughlin's results can be derived on the single assumption of central charge $c=1$. To this end we consider the bosonic representation of section 5 and we identify the operator content for a given $m$ as follows. We first notice that the Hall current for a state with filling fraction $\nu$ is

$$
J^{i}=\nu \frac{e}{2 \pi} \epsilon^{i j} E^{j} .
$$

Given our previous identification of the Hall current with the chiral anomaly of the CFT on the cylinder, we recognize that the factor $\nu$ has to appear also in the chiral anomaly equation

$$
\left(\partial_{t}+v \partial_{x}\right) \rho_{R}=\nu \frac{e}{2 \pi} E
$$

Comparing this with the bosonic representation (5.26) of this equation, we conclude that the effective CFT for the description of an incompressible state with filling fraction $\nu$ is given by (5.1) with

$$
\kappa=\frac{1}{\nu}=m
$$


We now explore the consequences of this identification.

First of all, we note that the Casimir energy $E_{0}=-v / 24 R$, and the corresponding quantum pressure $P=-v / 48 \pi R^{3}$ of the electron liquid depend on the filling fraction $\nu$ only through the velocities $v$. An independent measurement of $P$ and $v$ for various filling fractions $\left(\nu=1, \frac{1}{3}, \frac{1}{5}\right)$ would thus provide an experimental confirmation of the generic relationship between incompressibility and conformal invariance for these states.

Given the identification (6.3), we can recognize the excitations about the incompressible state of filling fraction $\nu=1 / m$ from the partition function (5.18) for $p=m, q=1$. These are neutral, gapless edge excitations (boundary phonons) and quasi-particle and quasi-hole excitations with charges $Q_{k}= \pm k / m$, and conformal dimensions $h_{k}=k^{2} / 2 m$, with $k$ integer. As in eq.(4.8), they yield the energy spectra

$$
\Delta E_{n, k}=\gamma(k)+\frac{v}{R} \frac{k^{2}}{2 m}+\frac{v n}{R} .
$$

The first term is the non-universal gap for charged excitations which can be again included by deforming the CFT as discussed in section 3 .

There are $2 m$ basic charged excitations associated to the primary fields $V_{ \pm 1}, \ldots, V_{ \pm m}$. Repeating the arguments of section 4, we conclude that the excitations about the incompressible ground state of filling $\nu=1 / m$ carry fractional charge

$$
Q_{k}= \pm \frac{k}{m}
$$

and fractional statistics

$$
\theta_{k}=\frac{k^{2}}{m} \bmod 2
$$

Since $m=$ odd integer, there is always the excitation $V_{m}$ with the quantum numbers $Q=1$ and $\theta=1$ of an electron. These results are in perfect agreement with Laughlin's theory, although they have been derived from a completely different point of view. Moreover, as already emphasized, conformal invariance leads to predictions on finite size effects, which are beyond the scope of the "plasma analogy", the main computational tool in Laughlin's theory. These predictions are the universal Casimir energy and the $1 / R$-corrections to the gaps for quasi-particle and quasi-hole excitations of eq.(6.4). Note that all these quantities have the same $(v / R)$-dependence. Therefore, the ratios $(1 / R$-parts)

$$
\frac{\Delta E_{0, k}}{E_{0}}=\frac{12 k^{2}}{m}, \quad \frac{\Delta E_{0, p}}{\Delta E_{0, q}}=\frac{p^{2}}{q^{2}}, \quad k, p, q=1, \ldots, m
$$


are universal quantities depending only on the filling fraction. A measurement of these quantities, either by direct experiment or by numerical simulation, would be a new and further confirmation of the ideas presented in this paper.

We thus conclude by stressing the importance of finite-size effects in the physics of the quantum Hall effect.

\section{Acknowledgments}

We thank Sergio Fubini for inspiration and constant encouragement. We also thank Luis Alvarez-Gaumé and Jürg Fröhlich for helpful discussions. G. R. Z. thanks the World Laboratory for partial support. G. V. D. gratefully acknowledges the support of the Mathematics Department and the Center for Theoretical Physics at MIT, where some of this work was done, and partial support through NSF grant 87-08447. 


\section{Appendix A.}

\section{Direct derivation of long range order on the boundary}

In section 4, we showed how conformal invariance implies algebraic long-range order in the density matrix for points $z_{1}, z_{2}$ on the boundary of the droplet. Here we present the direct microscopic derivation of this result for filling fraction $\nu=1$, thereby confirming the prediction of the CFT. Let us describe the steps of the derivation.

The density matrix is

$$
\rho\left(z_{1}, z_{2}\right)=\left\langle\Omega\left|\Psi^{\dagger}\left(z_{1}\right) \Psi\left(z_{2}\right)\right| \Omega\right\rangle, \quad \text { for } z_{1}=\operatorname{Re}^{i \theta_{1}}, z_{2}=\operatorname{Re}^{i \theta_{2}} .
$$

We let $R \rightarrow \infty$ together with the number of particles, $R^{2}=L+\mu$, taking for ease of computation $e B=2$ (i.e., $\ell=1$ ). The explicit computation is based on the steepestdescent approximation of the incomplete gamma function $\gamma(\alpha, x)$. One has

$$
\begin{aligned}
\rho\left(z_{1}, z_{2}\right) & =\frac{1}{\pi} \mathrm{e}^{-\frac{1}{2}\left(\left|z_{1}\right|^{2}+\left|z_{2}\right|^{2}\right)} \sum_{k=0}^{L} \frac{\left(\overline{z_{1}} z_{2}\right)^{k}}{k !}, \\
& =\frac{1}{\pi} \mathrm{e}^{R^{2}\left(e^{i \phi}-1\right)}\left[1-\frac{R^{2(L+1)}}{L !} \int_{0}^{e^{i \phi}} d t \mathrm{e}^{L(-t+\log t)} \mathrm{e}^{-\mu t}\right],
\end{aligned}
$$

where $\phi=\theta_{2}-\theta_{1}$, and the integral in the second term is taken along a ray from the origin to the point $e^{i \phi}$ in the complex $t$-plane. We used an integral representation of $\gamma\left(1+L, R^{2} e^{i \phi}\right)$, i.e., of the confluent hypergeometric function [27], and made some changes of variables. Following reference [39], the asymptotic expansion of the integral for $L \rightarrow \infty$, is obtained by deforming the integration contour along the steepest paths

$$
\operatorname{Im} h(t)=\operatorname{Im} h\left(t_{o}\right), \quad h(t)=-t+\log t, \quad t=x+i y,
$$

where $t_{o}$ is one of the two end-points of integration.

As a warming up exercise, let us first consider the case of the density $\rho(z)$, i.e., $z_{1}=z_{2}$, $\phi=0$ and also $\mu=0$. The saddle point $t=1$ is one integration end point, and the two steepest paths passing through it are the solutions of

$$
\operatorname{Im} h(t)=-y+\arctan \frac{y}{x}=0, \quad \rightarrow \quad x=\frac{y}{\tan y},
$$


namely, the line $y=0$ and a non-trivial curve for $x<1$, ending at $(x, y)=\left(0, \pm \frac{\pi}{2}\right)$. The integration path in eq. (A.2) coincides with the steepest path $y=0$, and it is not deformed in this case. The saddle-point expansion of the integrand $\exp h(t)$, including a factor $1 / 2$ for the half-path, gives

$$
\int_{0}^{1} d t e^{L(-t+\log t)} \sim \frac{1}{2} e^{-L}\left(\sqrt{\frac{2 \pi}{L}}+\frac{4}{3} \frac{1}{L}+O\left(\frac{1}{L \sqrt{L}}\right)\right)
$$

Thus,

$$
\rho(|z|=\sqrt{L})=\frac{1}{\pi}\left(\frac{1}{2}-\frac{2}{3} \frac{1}{\sqrt{2 \pi L}}+O\left(\frac{1}{L}\right)\right) .
$$

Actually, in the thermodynamic limit, the density of the droplet becomes a representation of the step function $\rho(z)=\frac{1}{\pi} \theta\left(L-|z|^{2}\right)$, whose mid-point regularized value is $1 / 2$.

In the case of the density matrix, $\phi \neq 0$, the steepest path going from $t=0$ to $t=e^{i \phi}$, is the unique solution to

$$
\operatorname{Im} h(t)=\operatorname{Im} h\left(e^{i \phi}\right) \quad \rightarrow \quad x=\frac{y}{\tan (y+\phi-\sin \phi)}
$$

This path lies in the region $x<1$, and connects the three points $(x, y)=\left(0, \frac{\pi}{2}-\phi+\sin \phi\right)$, $(\cos \phi, \sin \phi)$ and $(0,0)$; so it is conveniently parametrized by $y$. One can show that $R e h(t)$ is monotonically increasing in $y$, i.e., along the path. Therefore, after deforming the integral along this path, its asymptotic value is dominated by the integration region around the end-point $t=e^{i \phi}$, where $R e h(t=x(y)+i y)$ can be expanded to first order as

$$
\begin{aligned}
& \int_{0}^{e^{i \phi}} d t e^{L h(t)-\mu t} \\
& \quad \sim \exp \left(L\left(i \phi-e^{i \phi}\right)-\mu e^{i \phi}\right) \int_{-\infty}^{\sin \phi} d y e^{2 L(y-\sin \phi) \tan \frac{\phi}{2}}\left(\left(\frac{d x}{d y}\right)_{y=\sin \phi}+i\right) \\
& \quad=\exp \left(L\left(i \phi-e^{i \phi}\right)-\mu e^{i \phi}\right) \frac{1}{L} \frac{e^{i \phi}}{1-e^{i \phi}} .
\end{aligned}
$$

By collecting all factors, the asymptotic form of the density matrix (A.1) for points on the boundary is

$$
\left\langle\Omega\left|\Psi^{\dagger}\left(R e^{i \theta_{1}}\right) \Psi\left(R e^{i \theta_{2}}\right)\right| \Omega\right\rangle \sim \frac{1}{\pi} \frac{1}{\sqrt{2 \pi} R} e^{i\left(R^{2}-\mu\right)\left(\theta_{2}-\theta_{1}\right)} \frac{e^{i \theta_{2}}}{e^{i \theta_{2}}-e^{i \theta_{1}}}, \quad(R \rightarrow \infty) .
$$

Upon using $R^{2}-\mu=L$, this is in agreement with the prediction (4.12) of the CFT. 


\section{References}

[1] R. B. Laughlin, Phys. Rev. Lett. 50 (1983) 1395.

[2] R. E. Prange and S. M. Girvin eds., "The Quantum Hall effect", Springer, New York, (1990).

[3] Y. S. Wu, "Topological Aspects of the Quantum.Hall Effect", in "Physics, Geometry and Topology", H. C. Lee ed., Proc. Banff Conf. 1989, Plenum Press, New York (1990).

[4] E. Fradkin, "Field Theories of Condensed Matter systems", Addison-Wesley, New York, (1991).

[5] A. Cappelli, C. A. Trugenberger, and G. R. Zemba, "Infinite Symmetry in the Quantum Hall Effect", CERN preprint 6516/92.

[6] S. Iso, D. Karabali and B. Sakita, "Fermions in the Lowest Landau Level: Bosonization, $W_{\infty}$ Algebra, Droplets, Chiral Boson", CCNY-HEP-92/6 preprint; A. Dhar, G. Mandal and S. R. Wadia, "Classical Fermi Fluid and Geometrical Action for c=1", IASSNS-HEP-91/89 preprint.

[7] B. I. Halperin, Phys. Rev. B25 (1982) 2185.

[8] X. G. Wen, "Gapless Boundary Excitations in the Quantum Hall States and the Chiral Spin States", preprint NSF-ITP-89-157, Phys. Rev. Lett. 64 (1990) 2206; B. Block and X. G. Wen, Phys. Rev. B42 (1990) 8133; X. G. Wen, Phys. Rev. B41 (1990) 12838, D. H. Lee and W. G. Wen, Phis. Rev. Lett. 66 (1991) 1765.

[9] M. Stone, Ann. Phys. (NY) 207 (1991) 38, Phys. Rev. B42 (1990) 8399, Int. Jour. Mod. Phys. B 5 (1991) 509.

[10] J. Fröhlich and A. Zee, Nucl. Phys. B364 (1991) 517; J. Fröhlich and T. Kerler, Nucl. Phys. B 354 (1991) 369.

[11] For a review see: X. G. Wen, Int. Jour. Mod. Phys. B6 (1992) 1711.

[12] R. B. Laughlin, "Elementary Theory: the Incompressible Quantum Fluid", in [2].

[13] For a review see: F. Wilczek, "Fractional Statistics and Anyon Superconductivity", World Scientific, Singapore, (1990).

[14] R. B. Laughlin, "Fractional Statistics in the Quantum Hall effect", in [13].

[15] A. A. Belavin, A. M. Polyakov and A. B. Zamolodchikov, Nucl. Phys. B 241 (1984) 333.

[16] P. di Francesco, H. Saleur and J.-B. Zuber, J. Stat. Phys. 49 (1987) 57.

[17] S. M. Girvin and A. H. Mac Donald, Phys. Rev. Lett. 58 (1987) 1252; N. Read, Phys. Rev. Lett. 62 (1989) 86.

[18] For a review see: P. Ginsparg, "Applied Conformal Field Theory", in "Fields, Strings and Critical Phenomena", Les Houches 88, E. Brezin and J. Zinn-Justin eds., NorthHolland, Amsterdam (1990).

[19] R. Floreanini and R. Jackiw, Phys. Rev. Lett. 59 (1987) 1873. 
[20] J. L. Cardy, "Conformal Invariance and Statistical Mechanics", in "Fields, Strings and Critical Phenomena", Les Houches 88, E. Brezin and J. Zinn-Justin eds., NorthHolland, Amsterdam (1990).

[21] S. Fubini, Mod. Phys. Lett. A6 (1991) 347; S. Fubini and C. A. Lütken, Mod. Phys. Lett. A 6 (1991) 487.

[22] G. V. Dunne, A. Lerda and C. A. Trugenberger, Mod. Phys. Lett. A 6 (1991) 2819.

[23] C. Cristofano, G. Maiella, R. Musto and F. Nicodemi, Phys. Lett. B 262 (1991) 88, Mod. Phys. Lett. A 6 (1991) 1779, Mod. Phys. Lett. A 6 (1991) 2985, "The Quantum Hall Effect at Arbitrary Rational Filling: a proposal", Napoli preprint DSF-T-92/05.

[24] G. Moore and N. Read, Nucl. Phys. B 360 (1991) 362, "Non-abelions in the Fractional Quantum Hall Effect", proc. 4-th Yukawa Int. Seminar, Kyoto, July 1991, Prog. Theor. Phys. Supp. 107 (1992), Y. Nagaoka ed..

[25] X. G. Wen, Phys. Rev. B40 (1989) 7387, Int. Jour. Mod. Phys. B2 (1990) 239; X. G. Wen and Q. Niu, Phys. Rev. B41 (1990) 9377.

[26] R. Iengo and K. Lechner, Phys. Rep. C 213 (1992) 179.

[27] I. S. Gradshteyn and I. M. Ryzhik, "Table of Integrals, Series and Products", Academic Press, New York (1980).

[28] R. Jackiw and S.-Y. Pi, Phys. Rev. D42 (1990) 3500.

[29] E. Witten, Comm. Math. Phys. 121 (1989) 351.

[30] C. Itzykson and J. M. Drouffe, "Statistical Field Theory", Cambridge Univ. Press, Cambridge (1989), vol. 2, pages $540 \mathrm{ff}$.

[31] A. Cappelli, C. A. Trugenberger and G. R. Zemba, to appear.

[32] M. B. Green, J. H. Schwarz and E. Witten, Superstring Theory, Cambridge Univ. Press, Cambridge, (1988).

[33] F. D. M. Haldane and E. H . Rezayi, Phys. Rev. Lett. 54 (1985) 237; G. Fano, F. Ortolani and E. Colombo, Phys. Rev. B34 (1986) 2370.

[34] R. Morf and B. I. Halperin, Phys. Rev. B33 (1986) 2221; N. Datta and R. Ferrari, Investigations of the Laughlin State, Max-Planck preprint MPI-Ph/92-16.

[35] M. Stone, H. W. Wyld and R. L. Schult, "Edge Waves in the Quantum Hall Effect and Quantum Dots", preprint ILL(TH)-91-27.

[36] For a review see: S. B. Treiman, R. Jackiw, B. Zumino and E. Witten, Current Algebra and Anomalies, Princeton Univ. Press, New Jersey (1985).

[37] C. Imbimbo and A. Schwimmer, Phys. Lett. B193 (1987) 455.

[38] L. Faddeev and R. Jackiw, Phys. Rev. Lett. 60 (1988) 1692.

[39] A. Erdélyi, "Asymptotic Expansions", Dover, New York (1956). 


\section{Figure captions}

\section{Fig. 1}

The structure of the filled first Landau level in the disk geometry. All states up to angular momentum $L$ are occupied.

\section{Fig. 2}

The expectation value of the charge density in units $1 / \ell^{2}$ for the first Landau level filled up to $L=50$ as a function of $r / \ell$.

\section{Fig. 3}

The expectation value of the transverse current in units $1 /\left(-2 m \ell^{3}\right)$ as a function of $r / \ell$ for the first Landau level filled up to $L=50$.

\section{Fig. 4}

Typical one-particle spectrum for an annulus geometry of radii $R_{1} \simeq \ell \sqrt{L_{1}}$ and $R_{2} \simeq \ell \sqrt{L_{2}}$. Full dots denote occupied levels.

\section{Fig. 5}

Typical one-particle spectrum for the disk geometry of radius $R \simeq \ell \sqrt{L}$. See eq.(2.18).

\section{Fig. 6}

The one-body spectrum $\varepsilon_{l}$ in eq.(2.22) for $\Phi=10$.

\section{Fig. 7}

The conformal map from the Minkowskian cylinder $(R \theta, t)$ to the conformal plane $\eta$, see eq. (3.6). 